\title{
High Chromaticity Aluminum Plasmonic Pixels for Active Liquid Crystal Displays
}

\author{
Jana Olson ${ }^{a, b}$, Alejandro Manjavacas ${ }^{c}$, Tiyash Basu ${ }^{a, b}$, Da Huang ${ }^{a, b}$, Andrea E. Schlather $^{a, b}$, Bob Zheng $^{b, e}$, \\ Naomi Halas ${ }^{a, b, d, e}$, Peter Nordlander ${ }^{b, d}$, and Stephan Link ${ }^{* a, b, e}$ \\ ${ }^{a}$ Department of Chemistry, Rice University \\ ${ }^{b}$ Laboratory for Nanophotonics, Rice University \\ 'Department of Physics and Astronomy, University of New Mexico \\ ${ }^{\mathrm{d}}$ Department of Physics and Astronomy, Rice University \\ ${ }^{\mathrm{e}}$ Department of Electrical and Computer Engineering, Rice University \\ Rice University, 6100 Main Street, Houston TX 77005 \\ University of New Mexico, Albuquerque, New Mexico 87131, United States
}

Key Words: surface plasmon, diffractive coupling, Fano resonance, color, nanorod, nanoparticle arrays 


\begin{abstract}
Chromatic devices such as flat panel displays could, in principle, be substantially improved by incorporating aluminum plasmonic nanostructures instead of conventional chromophores that are susceptible to photo-bleaching. In nanostructure form, aluminum is capable of producing colors that span the visible region of the spectrum while contributing exceptional robustness, low cost, and streamlined manufacturability compatible with semiconductor manufacturing technology. However, individual aluminum nanostructures alone lack the vivid chromaticity of currently available chromophores because of the strong damping of the aluminum plasmon resonance in the visible region of the spectrum. In recent work, we showed that pixels formed by periodic arrays of Al nanostructures yield far more vivid coloration than the individual nanostructures. This progress was achieved by exploiting far-field diffractive coupling, which significantly suppresses the scattering response on the long-wavelength side of plasmonic pixel resonances. In the present work, we show that by utilizing another collective coupling effect, Fano interference, it is possible to substantially narrow the shortwavelength side of the pixel spectral response. Together, these two complementary effects provide unprecedented control of plasmonic pixel spectral lineshape, resulting in aluminum pixels with far more vivid, monochromatic coloration across the entire RGB color gamut than previously attainable. We further demonstrate that pixels designed in this manner can be used directly as switchable elements in liquid crystal displays and determine the minimum and optimal numbers of nanorods required in an array to achieve good color quality and intensity.
\end{abstract}


Metallic nanostructure-based coloration using the spectral tunability of localized surface plasmon resonances is becoming an increasingly popular subject of research. ${ }^{1}$ The plasmon resonance is a collective oscillation of the conduction band electrons, and is highly sensitive to the shape, size, and material of the nanostructure. ${ }^{2-4}$ Previously, semiconducting quantum dots with their quantum confinement-based luminescence were initially incorporated into existing display technology, ${ }^{5,6}$ but currently a variety of novel plasmon-based coloration schemes are in rapid development. To date, gold, ${ }^{7}$ silver, ${ }^{8-10}$ and aluminum ${ }^{11-16}$ nanostructures have shown significant potential in coloration-based applications. In particular, aluminum in nanostructured form has received much recent attention for several reasons: its plasmon resonance is tunable across the entire visible wavelength range, it is an inherently low-cost, sustainable material, and it is compatible with complementary metal-oxide semiconductor (CMOS) manufacturing techniques. ${ }^{15,17}$ These factors make aluminum particularly attractive for large-area technological applications, including solar cells, ${ }^{18,19}$ filters for color imaging, ${ }^{20-24}$ photodetectors, ${ }^{25}$ solid-state lighting components, ${ }^{26}$ and flat-panel displays. ${ }^{27,}{ }^{28}$ To construct plasmonic color devices, nanostructures are typically grouped into micron-scale arrays known as pixels. ${ }^{7,17,28}$ The shape of the constituent nanostructures, as well as their patterned arrangement (or lack thereof), can both be used to modify and improve pixel coloration properties. ${ }^{7,8,12,29}$ Examples include incorporation of Fabry-Perot resonances either within individual annular rings in an array $^{21}$ or between layers of silver metal with no structural patterning at all. ${ }^{20}$ The crystallinity of the aluminum nanostructure itself can also have a drastic effect on its color. ${ }^{30}$ Specific shaping of the individual structural elements or the use of different periodic geometries for nanostructure patterning provide additional parameters for designing color filters. ${ }^{31,32}$ Stretchable substrates ${ }^{33,34}$ or liquid crystals ${ }^{35}$ can even contribute to active control over the color of an array of nanoparticles with anisotropic response.

Aluminum as a material for plasmonic pixels offers some very compelling advantages: the potential for ultrasmall pixels that increase display resolution, as well as low-cost possibilities for large-area display systems. However, an intrinsic limitation of aluminum plasmon resonances in color devices is that they are spectrally broad. This is particularly true in the red region of the visible spectrum, where the interband transitions introduce significant nonradiative damping beyond $600 \mathrm{~nm}$. Nanoparticles with broad optical resonances appear muted with pastel-like colors rather that the bright, more monochromatic "vivid" colors associated with a narrow optical response. In a recent publication, we showed that for pixels composed of an array of oriented Aluminum nanorods, it is possible to introduce a cut-off on the red side of the scattering spectrum of a pixel by exploiting far-field diffractive coupling, 
resulting in a significant narrowing of its spectral response and significantly more monochromatic pixels. ${ }^{36}$ The spectral cut-off introduced by far-field diffractive coupling depends on the lattice constants of the array and can be tuned independently of the plasmon resonance of the individual nanorod elements which is controlled by their aspect ratio.

In this work, we employ the same type of pixels consisting of arrays of aluminum nanorods and show how another collective effect, specifically a Fano interference between the plasmon resonance of the nanostructure and the lattice modes of the array, can be exploited to narrow the blue side of the scattering spectrum of such a structure. While this effect also depends upon the lattice constants of the array, it can be tuned entirely independently of the diffractive coupling effect. The combined effect of these two tuning mechanisms is pixels with extremely vivid monochromatic colors across the full RGB color gamut. Arrays that exploit both effects have been fabricated with dimensions ranging from $1 \mu \mathrm{m} x$ $1 \mu \mathrm{m}$ to at least $1 \mathrm{~mm} \times 1 \mathrm{~mm}$ without significant changes to their spectrum. The rational color design of these pixels relies on both the single nanorod plasmon resonance, and on the inter-rod spacings in two directions within the array, thereby enabling diffractive coupling and supporting additional Fano resonances. Because the plasmonic pixels described here consist of parallel nanorods, the polarization sensitivity of the single nanorod plasmon is transferred to the whole pixel, as we demonstrate by incorporating a set of red, green, and blue pixels into a liquid crystal display and modulating their intensity on and off with an applied voltage.

\section{Results and Discussion}

The vivid colors generated by each of the plasmonic pixels described in this work are produced by light scattering from an approximately hexagonal array of oriented aluminum nanorods of the same dimension in each case, as shown in Figure 1. The color-defining physical parameters for each pixel are the distance between rows of nanorods in the $y$ direction $\left(D_{y}\right)$, the distance between nanorods along the $x$ direction $\left(D_{x}\right)$, as well as the length $(/)$ and width $(w)$ of each nanorod (Figure 1a top view). The height of the nanorods is $35 \mathrm{~nm}$ for all pixels, and all nanorod widths are designed to be $40 \mathrm{~nm}$ for compatibility with scalable fabrication methods, such as nanoimprint, ${ }^{35,37-42}$ laser interference, ${ }^{43-45}$ or

extreme ultraviolet lithography. ${ }^{46,47}$ All samples are prepared using standard e-beam lithography on an indium tin oxide (ITO) coated glass substrate, followed by the spincoating of a layer of polyimide (PI), as 
shown in the main portion of Figure 1a. After fabrication, the pixels are then spectrally characterized using dark field excitation with p-polarized light oriented along the longitudinal axis of the nanorods.

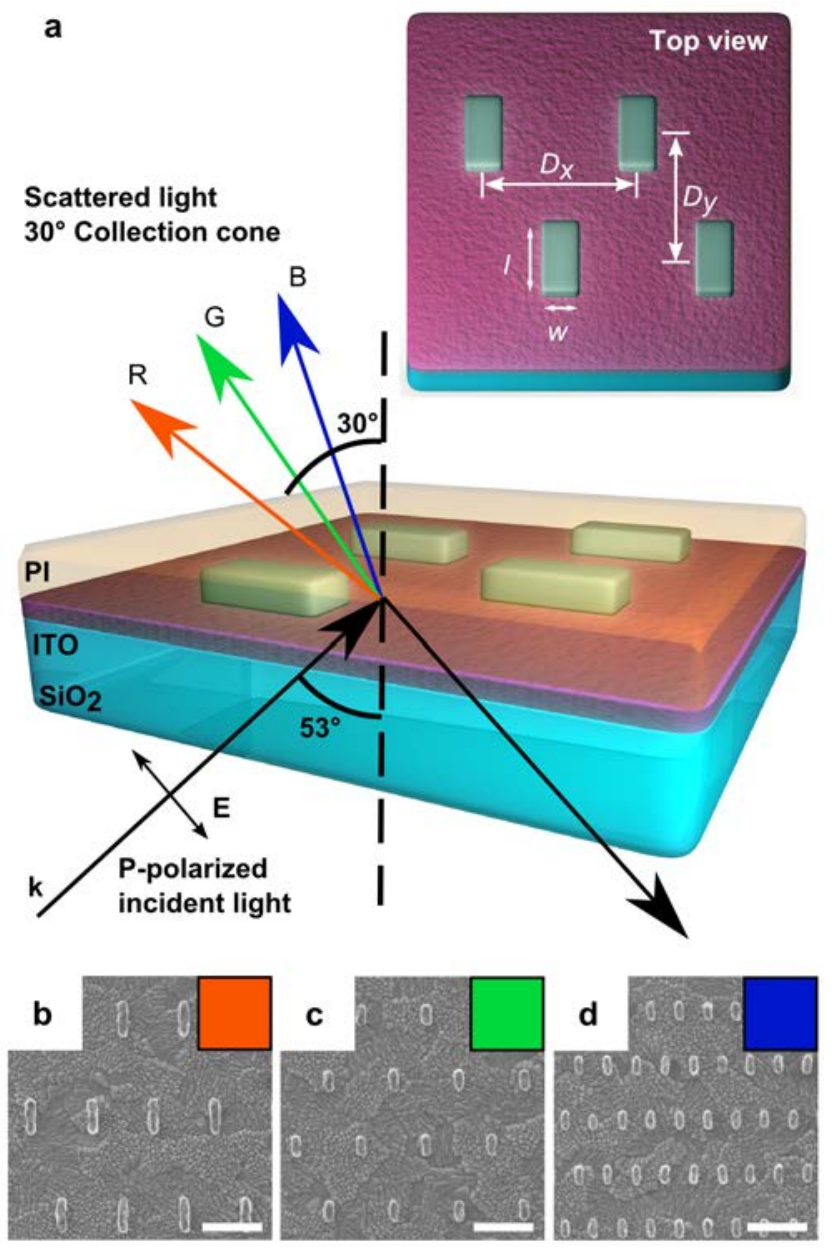

Figure 1. Plasmonic pixel fabrication. a, Inset: top down view of the layout of a plasmonic pixel, indicating the length $(I)$ and width $(w)$ of the nanorods and also the inter-rod spacings $\left(D_{x}\right.$ and $\left.D_{y}\right)$. The main panel of (a) shows the same pixel on an ITO-coated silica substrate prepared using e-beam lithography, then coated with a thin layer of PI. This sample is excited with p-polarized light in the pixel plane at an angle of $53^{\circ}$ normal to the glass surface, and the scattered light is collected from the top surface of the sample. $\mathbf{b}, \mathbf{c}$, and $\mathbf{d}$ show high-resolution SEM images of portions of a red $\left(I=153, D_{x}=\right.$ $\left.256, D_{y}=410\right)$, green $\left(I=88.5, D_{x}=272, D_{y}=272\right)$, and blue $\left(I=81, D_{x}=118, D_{y}=227\right)$ pixel, respectively, with marker bars indicating $250 \mathrm{~nm}$. The insets show the color created by a pixel of dimensions shown in each respective SEM image. All physical dimensions are in $\mathrm{nm}$. 
In general, longer nanorods and larger inter-rod distances produce redder colors, while shorter nanorods and smaller inter-rod distances produce bluer colors. Each individual pixel is designed to cover a footprint of $5 \mu \mathrm{m} \times 5 \mu \mathrm{m}$, unless otherwise stated. Figure $1 \mathrm{~b}$ is an SEM image showing a 1 micron region of a red pixel with nanorod length $I=153 \mathrm{~nm}, D_{x}=256 \mathrm{~nm}$, and $D_{y}=410 \mathrm{~nm}$. The color produced by this combination of physical parameters is orange-red (inset of Figure 1b), when measured using the dark field microscope described in the Methods section. Corresponding images for a green pixel $(I=89$ $\left.\mathrm{nm}, D_{x}=202 \mathrm{~nm}, D_{y}=272 \mathrm{~nm}\right)$ and a blue pixel $\left(I=81 \mathrm{~nm}, D_{x}=118 \mathrm{~nm}, D_{y}=227 \mathrm{~nm}\right)$ are given in Figures $1 \mathrm{c}$ and $1 \mathrm{~d}$, respectively.

The geometrical structure and thus the color of a plasmonic pixel is designed through theoretical considerations and simulated spectra based on a coupled dipole model, where all physical parameters of the experiment are explicitly considered (see Methods section for further details). The design process begins with the selection of a desired color and spectral shape, followed by optimization of $D_{y}$ to produce an appropriate threshold on the red side of the spectrum. Each pixel can be approximated by an array of point dipoles, and therefore behaves analogously to a grating that is excited at a non-normal angle of incidence. The threshold on the red side of the spectrum can then be described by: ${ }^{36}$

$$
\lambda_{\max }=D_{y} n\left(\sin \theta_{\text {incident }}+\sin \theta_{\text {observed }}\right)
$$

Here, $\lambda_{\max }$ is the maximum wavelength above which no constructive interference for the scattering by the pixel can be observed. The average refractive index $n$ is $1.55, \theta_{\text {incident }}$ is the angle at which the light is incident on the pixels, and $\theta_{\text {observed }}$ is the half-angle of the objective collecting the light. The incident light is coupled into the sample using a $60^{\circ}$ prism, and passes through layers of glass, ITO, and $\mathrm{PI}$, and therefore $\theta_{\text {incident }}=53^{\circ}$ inside the PI where the pixel is located, based on Snell's law. For an objective with a numerical aperture of NA $=0.8$, the observation angle is $30^{\circ}$ inside the $\mathrm{PI}$. These excitation and collection conditions apply for all experimental spectra and images reported here (measurements obtained with a dark field microscope) and are also included in the corresponding calculations. Figure $2 \mathrm{a}$ shows a series of simulated spectra for pixels with $I=85 \mathrm{~nm}$ and $D_{x}=280 \mathrm{~nm}$ as $D_{y}$ is increased from 200 $\mathrm{nm}$ through $340 \mathrm{~nm}$, with the dashed line indicating the calculated location of $\lambda_{\max }$ obtained from eq. (1) for each simulated spectrum. The analytical expression (eq. (1)) and the simulated spectra are in excellent agreement, and demonstrate that the threshold wavelength, above which destructive interference suppresses scattering, increases linearly with $D_{y}$. 

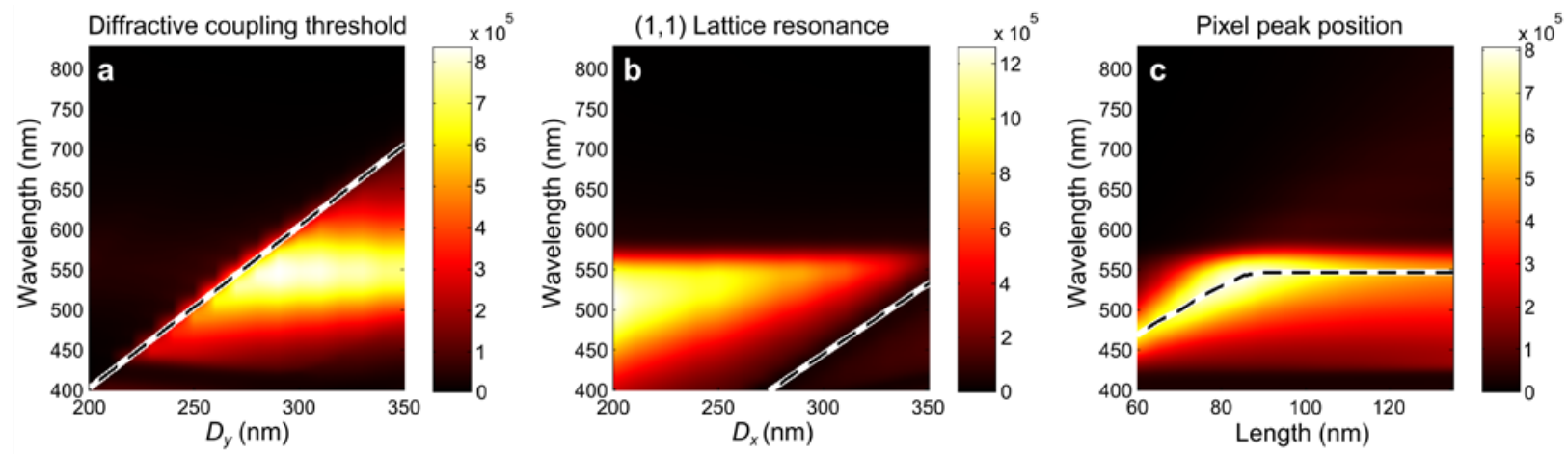

Figure 2. Theoretical basis for the three-step color design method. Calculated spectra are shown with intensity (color) as a function of wavelength (vertical axis). a, A series of spectra plotted as a function of $D_{y}$ varying over $200 \mathrm{~nm}$ to $350 \mathrm{~nm}$ in $10 \mathrm{~nm}$ increments while $D_{x}(280 \mathrm{~nm})$ and nanorod length $(85 \mathrm{~nm})$ are constant. The dashed line shows the positive linear relationship between the diffractive coupling threshold and the value of $D_{y}$, according to eq. (1). $\mathbf{b}$, A series of spectra plotted as a function of $D_{x}$ varying over $200 \mathrm{~nm}$ to $350 \mathrm{~nm}$ in $10 \mathrm{~nm}$ increments while $D_{y}(280 \mathrm{~nm})$ and nanorod length (85 nm) are constant. The dashed line shows the positive linear relationship between the $(1,1)$ grating resonance and the value of $D_{x}$, according to eq. (2). c, A series of spectra plotted as a function of nanorod length varying over 60 to $135 \mathrm{~nm}$ in $5 \mathrm{~nm}$ increments while $D_{x}(280 \mathrm{~nm})$ and $D_{y}(280 \mathrm{~nm})$ are constant. The dashed line shows the relationship between the pixel peak intensity and the length of the nanorod. In practice, the desired threshold is selected from step $a$, and then steps $b$ and $c$ are reiterated as necessary to obtain the desired color.

The second step in the design of the plasmonic pixel color is to select $D_{x}$ such that the combination of $D_{x}$ and $D_{y}$ together will produce one or more Fano resonances at locations in the pixel spectrum that will result in a narrowing of the spectral response. In the simplest case, the intensity dip associated with these resonances will act as a threshold on the blue side of the scattering spectrum. These Fano resonances are a well-known phenomenon, ${ }^{48-50}$ originating from the coupling between the broad dipole resonance of the individual nanorod and dark sharp lattice resonances of the array. Therefore, we can choose the spectral positions of different Fano resonances by selecting the suitable wavelengths of the lattice resonances, which are given by the following expression: ${ }^{48,50}$

$$
\lambda=2 \pi n /\left|\mathbf{k}_{||}-\mathbf{g}\right|
$$


$\mathbf{k}_{\|}$is the component of the wave vector of the incident light parallel to the array plane, and $\mathbf{g}$ is a reciprocal lattice vector (see the Methods section for more details). Here, we will exploit the Fano resonance associated with the following reciprocal lattice vector:

$$
\mathbf{g}_{(1,1)}=\left(2 \pi / D_{x}\right) \hat{\mathbf{x}}+\left(\pi / D_{y}\right) \widehat{\mathbf{y}}
$$

The unit vectors $\hat{\mathbf{x}}$ and $\hat{\mathbf{y}}$ are parallel to the short and long axes of the nanorod, respectively.

Figure $2 \mathrm{~b}$ shows simulated spectra as $D_{x}$ is varied from $200 \mathrm{~nm}$ to $340 \mathrm{~nm}$ for pixels with constant values of $I=85 \mathrm{~nm}$ and $D_{y}=280 \mathrm{~nm}$. The dashed line indicates the position of the $(1,1)$ lattice resonance calculated from eq. (2). A linear red-shift with $D_{x}$ is clearly visible from both simulated spectra and Eq. (2). For spectrally narrow (monochromatic) pixel colors, it is important to choose $D_{x}$ such that no lattice resonances overlap the center of the intended spectrum. For strong spectral overlap between the nanorod and lattice modes, a Fano dip would be induced which would distort the color of the pixel.

The third step in the design of a plasmonic color pixel is to select a nanorod aspect ratio that will produce a peak in the pixel spectrum at the desired location between the two thresholds imposed by $D_{y}$ and $D_{x}$. The simulated spectra in Figure $2 \mathrm{c}$ show how the peak intensity of a pixel with $D_{x}=280 \mathrm{~nm}, D_{y}=$ $280 \mathrm{~nm}$ redshifts with increasing nanorod length, as indicated by the dashed line. Rather than continuing to increase, however, the wavelength of the peak maximum is limited by the threshold imposed by $D_{y}$. In particular for these pixels, the redshift ceases at approximately $I=85 \mathrm{~nm}$, caused by the red-wavelength threshold at $563 \mathrm{~nm}$ imposed by $D_{y}$. As the nanorod length is further increased, the scattering intensity is reduced.

This 3-step design approach leads to pixels with intensely vivid colors that span the entire color gamut of visible light, as shown in Figure 3. The colors and spectra reported in Figure 3 originate from scattering by $5 \mu \mathrm{m} \times 5 \mu \mathrm{m}$ plasmonic pixels with optimized structural parameters, recorded under dark field excitation in an optical microscope (see Methods section). In Figure 3a, a color space diagram, based on the 1931 standard observer defined by the International Commission on Illumination (CIE), illustrates the range of colors visible to the human eye inside the colored region. The curved axis indicates the wavelengths in $\mathrm{nm}$ that correspond to each color, where the most vivid colors lie along the edge of the graph while pastel colors lie closer to the white point at $(0.33,0.33)$. The white triangles 
represent the red, green, and blue tristimulus values common to all standard red, green and blue (sRGB) displays. The white line connecting the SRGB values encloses the SRGB color gamut, the area of which correlates to the number of colors that can be produced by such a display. Standard displays incorporate red, green, and blue pixels while all other displayed colors result from superpositions of these three colors. The three white squares circled in Figure 3a represent colors produced by aluminum pixels where the design goal was to generate colors comparable to the sRGB values. The colors shown are calculated from measured dark field scattering spectra, which are plotted in Figure 3b with black arrows indicating the corresponding data. This set of aluminum pixels can be used to produce an aluminum plasmonic pixel color gamut spanning 59\% of the area of the sRGB color gamut. The efficiency defined as incident vs. scattered light intensity of a green pixel is estimated to be about $4 \%$ (see Supporting Information Section 2). It is important to note that this efficiency is only for the forward scattered light of a certain diffraction order and is furthermore estimated for a unit area of the pixel. Only $4 \%$ of this area is occupied by aluminum, but the space between the nanorods matters as well as it facilitates diffractive coupling. The efficiency could be increased by incorporating reflectors so that the excitation light is back-reflected into the device and light scattered by the pixel in the backward direction is collected as well. 

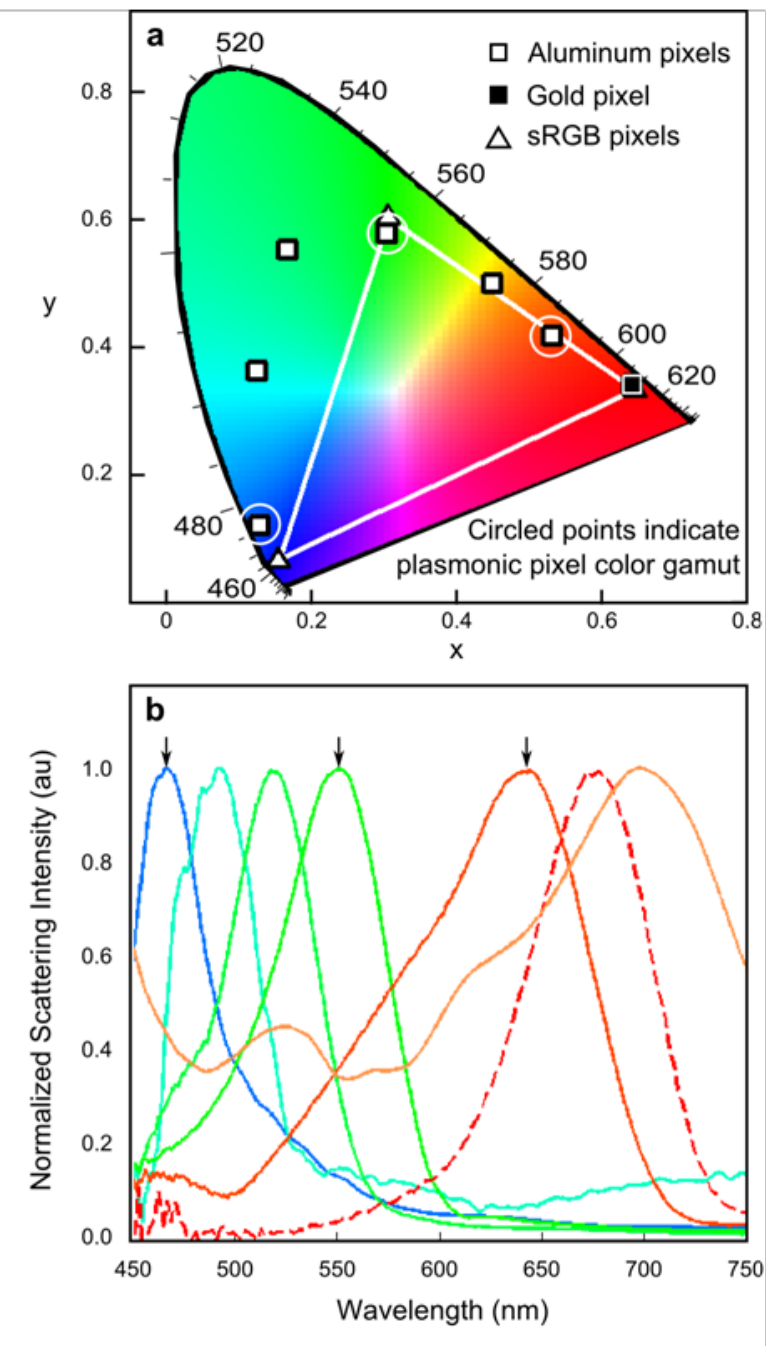

Figure 3. Color range of plasmonic pixels. a, The CIE color diagram, with color associated with every $(x, y)$ color coordinate pair plotted in the background of the figure. The color space is bounded by a curved axis denoting the wavelength of light (in $\mathrm{nm}$ ). The sRGB color gamut tristimulus values (white triangles) are connected with white lines. Several white squares show the $(x, y)$ color coordinates achieved by aluminum plasmonic pixels of different physical parameters. From blue to red, exact physical parameters measured in $\mathrm{nm}$ are $\left(I=116, w=70, D_{x}=297, D_{y}=348\right),\left(I=85, w=47, D_{x}=210, D_{y}=212\right)$, $\left(I=95, w=54, D_{x}=128, D_{y}=237\right),\left(I=180, w=49, D_{x}=288, D_{y}=322\right),\left(I=176, w=44, D_{x}=227, D_{y}=\right.$ $323),\left(I=100, w=43, D_{x}=272, D_{y}=271\right)$. The $(x, y)$ coordinates are $(0.44,0.50),(0.12,0.36),(0.12,0.12)$, $(0.53,0.41),(0.16,0.55),(0.30,0.58)$, respectively. The three circled points indicate a possible tristimulusbased color gamut using aluminum plasmonic pixels. The black square shows the $(x, y)$ color coordinates $(0.64,0.34)$ achieved by a gold plasmonic pixel of physical parameters in $\mathrm{nm}\left(I=57, w=30, D_{x}=346, D_{y}=\right.$ 
335). b, Spectra used to produce the calculated color coordinates in (a). The three black arrows indicate the three spectra associated with the red, green, and blue tristimulus values for the aluminum pixels. The dashed line indicates the spectrum of gold pixel. Each pixel had a footprint of $5 \mu \mathrm{m} \times 5 \mu \mathrm{m}$.

The introduction of additional plasmonic materials or multistimulus gamuts can further increase the area of the color gamut. If gold nanorods are used as the fundamental building block, a more vivid red plasmonic pixel can be achieved (black square in Figure 3a, dashed red line in Figure 3b). This gold pixel overlaps completely with the SRGB red tristimulus value. By using the blue and green aluminum pixels and the red gold pixel we can realize at least $88 \%$ of the SRGB gamut area. If a multi-stimulus color gamut is defined to include other experimental aluminum pixels with colors outside the SRGB gamut area (the two other white squares without a circle in Figure 3a and correspondingly color-coded spectra in Figure $3 \mathrm{~b}$ ), the area of the gamut increases to at least $92 \%$ of the SRGB color gamut and becomes $102 \%$ if the gold-based red pixel is included. Under this gamut definition, the blue-green region of the color gamut is enhanced beyond the capabilities of SRGB displays while maintaining most of the shared red and violet region.

Although the $5 \mu \mathrm{m} \times 5 \mu \mathrm{m}$ pixels discussed so far are smaller than the human eye can see, they can be tiled to cover a mm-scale area to produce colors bright enough to be seen easily by the naked eye, while maintaining the vivid color. Figure 4a shows a composite digital camera photograph of large area (1.5 $x 1.5 \mathrm{~mm}$ ) red, green, and blue pixels taken outside the microscope under edge-illumination by directing white light onto the edge of the slide, as illustrated in Figure $4 b$ (for more details see Supporting Information). The incident white light is polarized perpendicular to the substrate. Because of total internal reflection while the light travels through the glass substrate waveguide, a similar excitation geometry as in the dark field microscopy measurements is created, with the main difference being that the allowed excitation angles are larger and constrained by total internal reflection rather than the prism. According to Eq. (1), an increase in $\theta_{\text {incident }}$ for these pixels will result in a red shift in the wavelengths that can be collected over the collection cone defined by $\theta_{\text {observed. }}$ Therefore, for ideal color production, these pixels should be tailored to the excitation geometry under which they will be utilized. Eq. (1) further shows that the color of the pixels will depend on the viewing angle, which can impact realistic applications dependent on a wide range of viewing angles. However, the angle dependence can 
be mitigated with the help of a diffuser as Figure S2 and videos S1 and S2 in the Supporting Information clearly show.

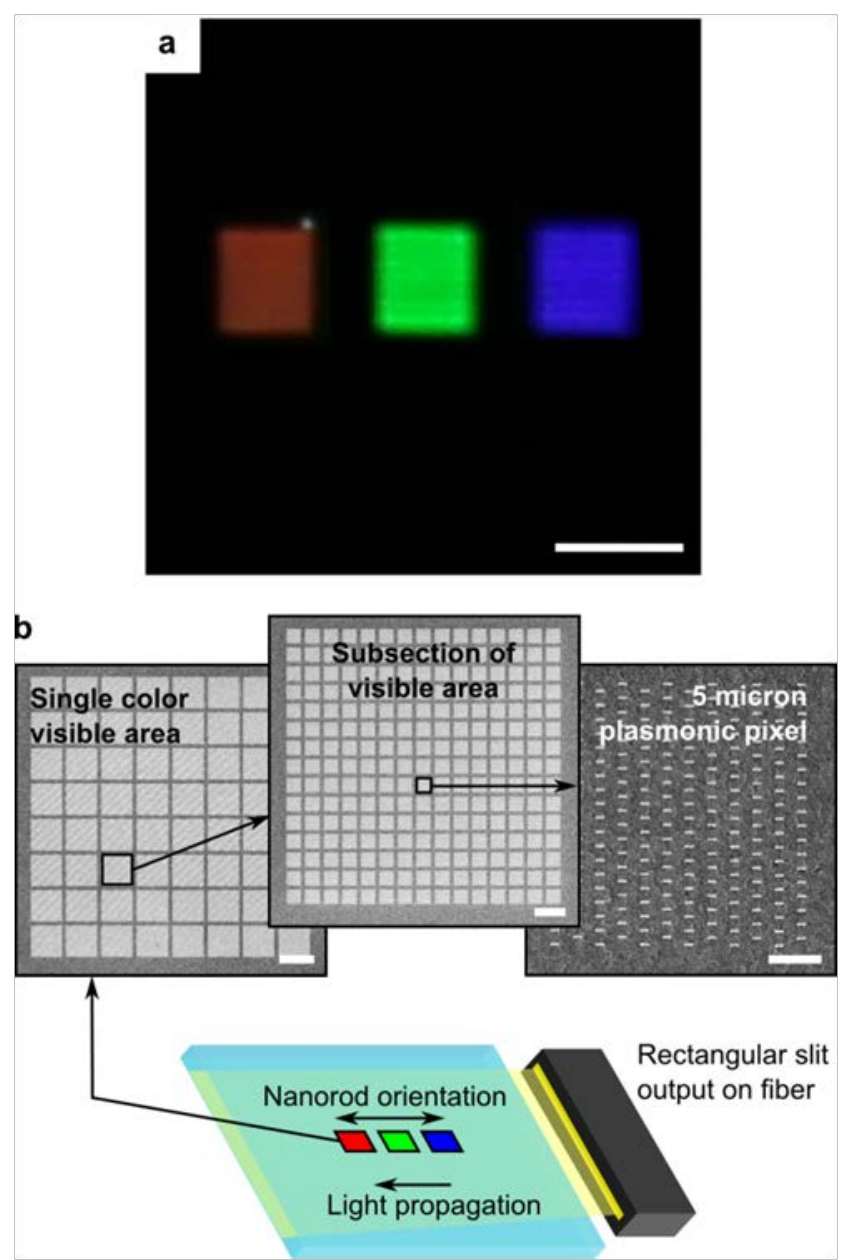

Figure 4. Scale-up into large area pixels from $5 \mu \mathrm{m} \times 5 \mu \mathrm{m}$ subpixels. a, Photograph of three $1.5 \mathrm{~mm} \times 1.5$ $\mathrm{mm}$ pixels as viewed outside the microscope. To most accurately represent the colors as viewed by the human eye, the pixels are shown in a composite image consisting of two images: the red and blue pixels are shown with $1 / 10 \mathrm{~s}$ exposure, while the green pixel is shown with $1 / 30 \mathrm{~s}$ exposure. These pixels are fabricated by e-beam lithography according to the scheme in (b). The measured dimensions of the pixels are (red: $w=47 \mathrm{~nm}, I=153 \mathrm{~nm}, D_{x}=257 \mathrm{~nm}, D_{y}=411 \mathrm{~nm}$ ), (green: $w=46 \mathrm{~nm}, I=89 \mathrm{~nm}, D_{x}=272 \mathrm{~nm}$, $D_{y}=272 \mathrm{~nm}$ ), and (blue: $w=40 \mathrm{~nm}, I=81 \mathrm{~nm}, D_{x}=119 \mathrm{~nm}, D_{y}=227 \mathrm{~nm}$ ). b, Large area pixels with footprints on the order of square $\mathrm{mm}$ are illuminated from the side, with the light traveling along the nanorods' long axes (bottom scheme). The light is carried by a fiber with a rectangular output, passed through a polarizer, and illuminated on the $1 \mathrm{~mm}$ side of the glass slide approximately normal to the 
edge surface. Each large colored region is composed of a tiling of $5 \mu \mathrm{m} \times 5 \mu \mathrm{m}$ pixels in the following layout. The $1.5 \times 1.5 \mathrm{~mm}$ area (top left) contains $8 \times 8$ subregions; each of these subregions (top center) is approximately $100 \mu \mathrm{m} \times 100 \mu \mathrm{m}$, and contains an array of $15 \times 15$ of the standard $5 \mu \mathrm{m} \times 5 \mu \mathrm{m}$ pixels (top right). The marker bars, from left to right, represent $100 \mu \mathrm{m}, 10 \mu \mathrm{m}$, and $1 \mu \mathrm{m}$. These SEM images have been contrast-enhanced.

Each of the $1.5 \times 1.5 \mathrm{~mm}$ color pixels in Figure $4 \mathrm{a}$ is composed of a tiling of smaller $5 \mu \mathrm{m} \times 5 \mu \mathrm{m}$ pixels as illustrated with the large-area red pixel in Figure $4 \mathrm{~b}$. The $1.5 \mathrm{~mm} \times 1.5 \mathrm{~mm}$ region is formed from $8 \mathrm{x}$ 8 sub-regions, and each of those $100 \mu$ m-wide sub-regions contains a $15 \times 15$ array of the originally designed $5 \mu \mathrm{m} \times 5 \mu \mathrm{m}$ pixels. The fundamental $5 \mu \mathrm{m} \times 5 \mu \mathrm{m}$ building blocks are the same as shown in the SEM images in Figures $1 \mathrm{~b}-\mathrm{d}$. These results demonstrate both that plasmonic pixels can be fabricated over large areas (total patterned footprint in Figure 4 a is $6.75 \mathrm{~mm}^{2}$ ), and that they are compatible with multiple excitation methods.

The nanorods that comprise the plasmonic pixels impart their polarization sensitivity to the entire patterned region, allowing the same pixels from Figure 4a to be incorporated into a LCD for complete on/off switching without significant change in color. Figure 5a shows a photograph of large-area red, green, and blue pixels incorporated into an LCD, which is prepared by placing the pixel substrate face to face with a similar substrate without pixels, where the ITO/PI surfaces are separated by approximately 6 $\mu \mathrm{m}$ of liquid crystal. The liquid crystal 4-Cyano-4'pentylbiphenyl (5CB) is aligned in a twisted nematic configuration, causing the scattered light from the pixels to be rotated by $90^{\circ}$ as it passes through the liquid crystal. A polarizer placed before the digital camera is orientated so that in this voltage-off state the pixels are visible (Figure 5b, top). When a voltage is applied across the $6 \mu \mathrm{m}$ gap, the anisotropic liquid crystal molecules realign parallel to the electric field and hence parallel to the direction of light propagation through the LCD. In this voltage-on state, the liquid crystal does not appreciably rotate the polarization of the light scattered by the pixels, and the polarizer thus blocks the light making the pixels invisible (Figure 5b, bottom). A video demonstrating this switching is available (Supporting Information Video S1). 


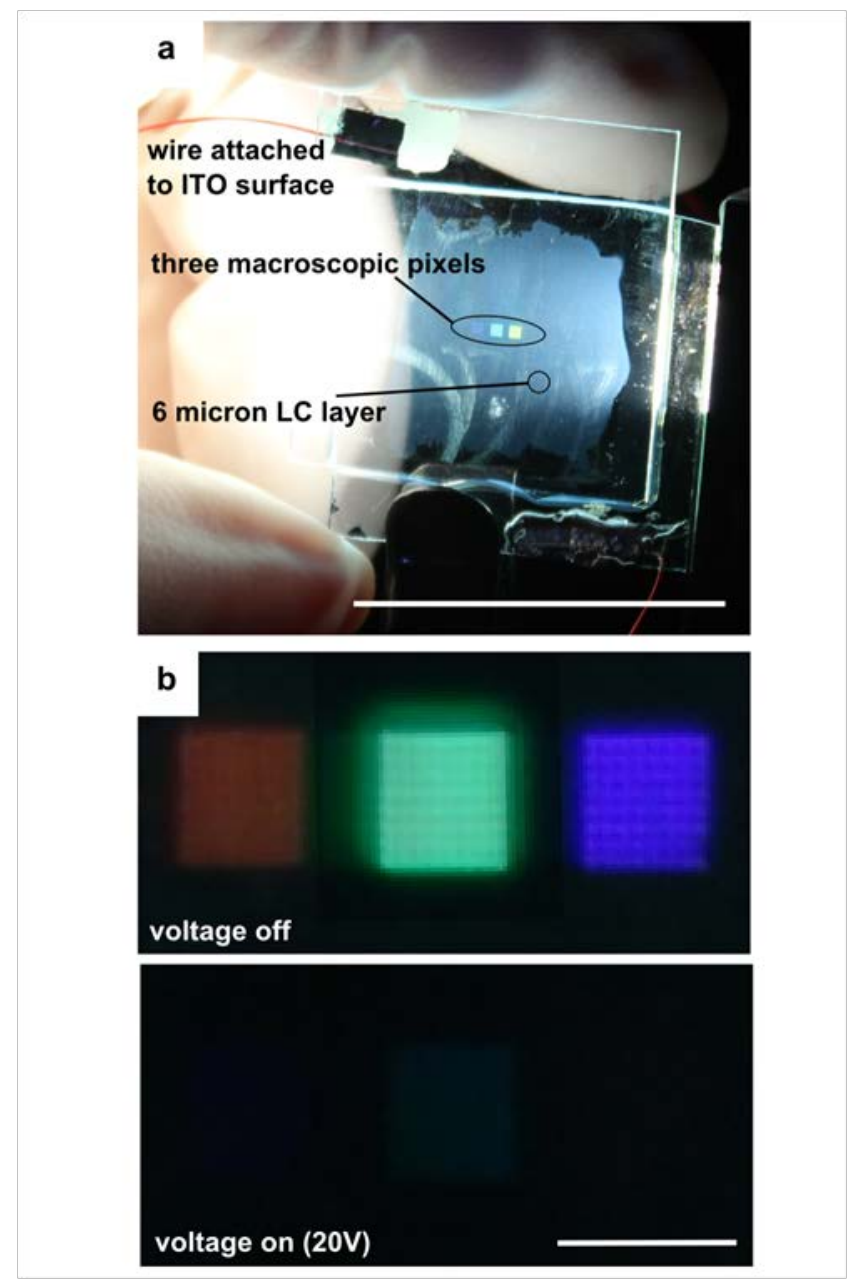

Figure 5. Liquid crystal-based on/off switching of large area plasmonic pixels. a, Digital camera image (straight-on view) of the same red, green, and blue large area pixel from Figure 4 in a LCD, held in-hand under white light edge-illumination with relevant components indicated in the figure. Marker bar indicates $2.5 \mathrm{~cm}$. b. Digital camera image of the on/off switching for these pixels in the LCD. In both the top and bottom of (b), the excitation geometry is the same as in Figure 4 and an additional polarizer is placed in front of the camera and set to section the polarization perpendicular to the nanorod long axis (horizontal in this image). This geometry is comparable to a commercial twisted nematic LCD. The twisted nematic liquid crystal rotates the scattered light polarization by 90 in the voltage off state and has no effect in the voltage on state. The top image is shown with no voltage applied, and the bottom image is shown with a $20 \mathrm{~V}, 10 \mathrm{kHz}$ square wave AC voltage applied. As in Figure 4a, the top image is a composite with the same exposure times as given for Figure 4a. The exposure time for the bottom image is $1 / 10 \mathrm{~s}$. These images are taken with the camera positioned at $30^{\circ}$ relative to the surface normal 
as most light is scattered in this directions. The marker bar represents $2 \mathrm{~mm}$, and applies to both images in (b).

The two effects that are used to sharpen the scattering spectra of the Al pixels, diffractive far-field coupling and Fano interference, are collective in nature and it is therefore of interest to investigate how the pixel response depend on the array size. Figure 6a shows the green portion of the color space diagram comparing the average color of seventeen 1-nanorod pixels (white marker), seven 9-nanorod pixels (black marker), fourteen 100-nanorod pixels (pink marker), and six 3969-nanorod pixels (blue marker). Multiple pixels were measured for each size to account for sample to sample heterogeneity for the same pixel design parameters. The single nanorod pixels were measured with a larger sample size because we observed larger spectral variations likely due to small nanorod size differences that are no longer averaged over in the single nanorod case. In each case, the average color of the four pixel sizes is located at the center of each crosshair, and the standard deviations in color coordinates $x$ and $y$ are plotted as the arm lengths of each crosshair, with all individual data points given in Figure S5. The average length of the nanorods for all samples is $I=85 \mathrm{~nm}$ with $D_{x}=317 \mathrm{~nm}$ and $D_{y}=317 \mathrm{~nm}$ for the pixels containing more than one nanorod. The 9-nanorod pixels, as well as even larger pixels, display significantly less color variation compared to the 1-nanorod pixels. The length and width of the e-beam lithographically prepared nanorods deviates from particle to particle by approximately $5 \%$, causing the aspect ratio to vary between 1.9 and 2.3. This variation drastically affects the color generated by an individual nanorod. When several nanorods are grouped into a pixel, these fluctuations due to size variations become less pronounced and diffractive coupling furthermore shapes the collective scattering spectrum. Surprisingly, it takes very few nanorods to generate reproducible colors. Already the 9nanorod pixel spectrum is very similar from sample to sample. These 9-nanorod pixels have a footprint of only about $1 \mu \mathrm{m} \times 1 \mu \mathrm{m}$. Larger pixels exhibit even smaller sample-to-sample variation. 

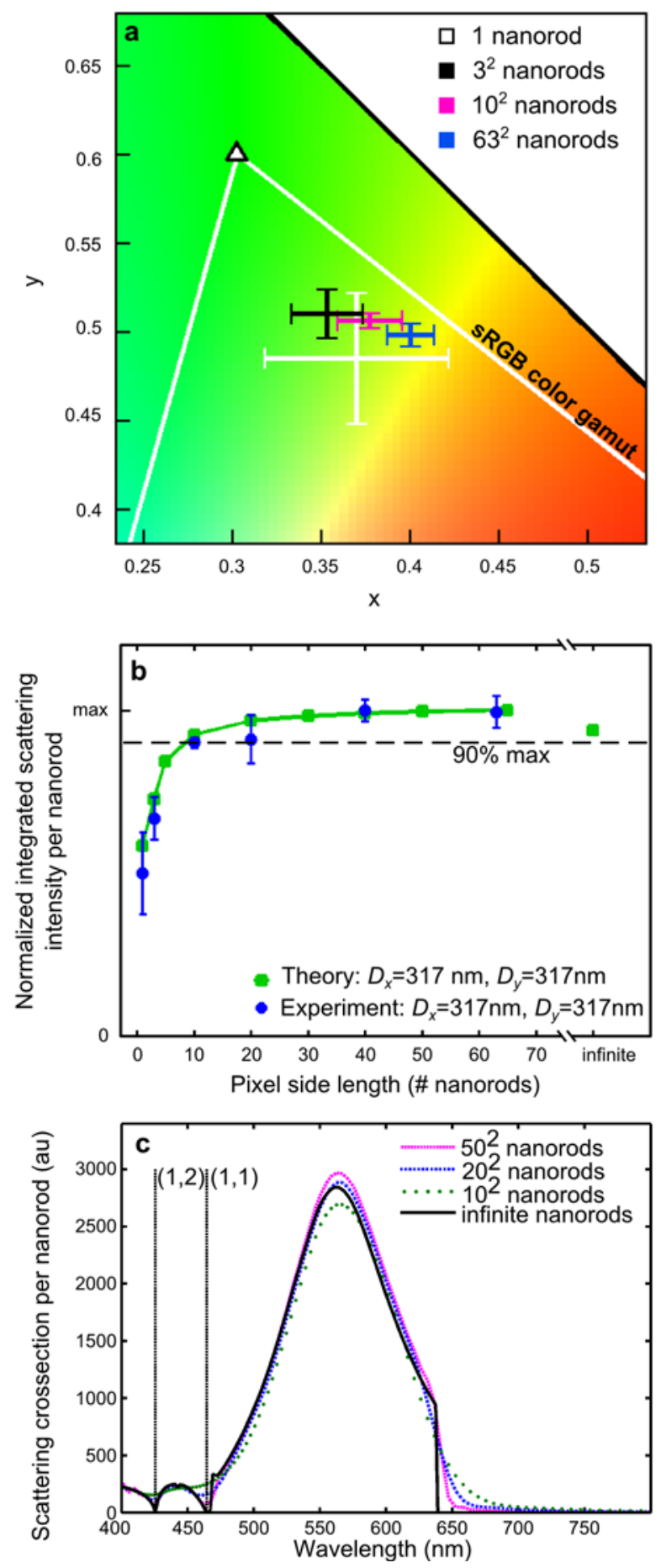

Figure 6. Color control over various pixel sizes. a, CIE diagram focusing on the green region of the visible color space. Seventeen single nanorods of average length $85 \mathrm{~nm}$ and average width $43 \mathrm{~nm}$ were measured under dark field excitation, with a white cross centered at the average $(x, y)$ coordinate and the standard deviation over those seventeen measurements shown as the length of the crosshair in $x$ 
and $y$. Seven $3 \times 3$-nanorod pixels of the same sized nanorods with $\left(D_{x}=317 \mathrm{~nm}, D_{y}=317 \mathrm{~nm}\right)$ (black cross), fourteen 10x10 nanorod pixels (pink cross), and six 63×63-nanorod pixels (blue cross) are also plotted. For reference, the SRGB color gamut in this region is also plotted. $\mathbf{b}$, Normalized scattering intensity per nanorod is plotted for pixels of increasing sizes, from a single nanorod to $65 \times 65$ nanorods per pixel. The same is also calculated for a pixel composed of infinite nanorods. Theoretical values for pixels with dimensions in $\mathrm{nm}\left(I=85, w=40, D_{x}=317, D_{y}=317\right)$ are plotted as squares, while experimental data points for pixels with dimensions in $\left(I=85, w=43, D_{x}=317, D_{y}=317\right)$ are given by circles, with error bars indicating the standard deviation over the same number of pixels as listed for (a). A dashed line indicates the point on the intensity axis that corresponds to $90 \%$ of the maximum intensity achieved with a pixel containing $65 \times 65$ nanorods. c, Selected calculated scattering spectra corresponding to the data in (b). The green dotted line represents a pixel containing 100 nanorods, the blue dashed-dotted line indicates a pixel of 400 nanorods, and the pink dashed line corresponds to a pixel of 2500 nanorods. The black solid line represents a pixel with an infinite number of nanorods. The two vertical black dashed lines point to the wavelength at which the $(1,2)$ and $(1,1)$ grating resonances occur: $426 \mathrm{~nm}$ and $465 \mathrm{~nm}$, respectively.

The cut-off thresholds on the red and blue sides of the spectrum imposed by collective effects play a significant role for the color control of the pixels, but the pixel does not need to cover a large area for these array-based collective effects to appear. We compare in Figure $6 \mathrm{~b}$ the integrated spectral intensities of pixels of increasing size: 1 nanorod through $65 \times 65$ nanorods. Experimentally, the integrated intensity was measured by imaging each pixel on a CCD camera and finding the total intensity of the pixel image. As the experimental measurements were carried out on multiple samples, the standard deviations account for sample heterogeneity that arises from the fabrication process. The nonmonotonic trend in error bars reflects this heterogeneity and also indicates limited sampling. In the theoretical calculations, the integrated intensity is equal to the sum of the spectral intensities. The dashed line in Figure $6 \mathrm{~b}$ indicates $90 \%$ of the normalized integrated scattering intensity per nanorod. This value is already reached for a pixel of $10 \times 10$ nanorods. We also note that it is not the ideal, infinitely large pixel that provides the greatest intensity per nanorod; pixels of finite size yield a greater integrated intensity per nanorod than an infinite pixel, as also seen from Figure 6b. The infinite pixel produces an integrated intensity per nanorod roughly equal to that of the $10 \times 10$ nanorod pixel. 
The scattering spectra of several simulated pixels from Figure $6 \mathrm{~b}$ are plotted in Figure $6 \mathrm{c}$. The spectra display Fano resonances located at $426 \mathrm{~nm}$ caused by the $(1,2)$ lattice resonance, and at $465 \mathrm{~nm}$ caused by the $(1,1)$ lattice resonance. Both lattice resonances are indicated via vertical lines in Figure $6 \mathrm{c}$. As the number of nanorods per pixel increases from $10^{2}$ to $50^{2}$ nanorods, the total intensity of the pixel increases. As expected, an increase in the number of nanorods in the array produces sharper features at the Fano resonances and at the far-field coupling threshold at $639 \mathrm{~nm}$. However, the infinite pixel does not exhibit the greatest scattering cross section per nanorod, as already demonstrated by the data in Figure $6 \mathrm{~b}$. The most likely explanation is that the nanorods at the edges of finitely-sized pixels scatter light more efficiently to the far-field, contributing significantly to the signal in smaller sized pixels (Figure S6 and S7). Therefore, the best choice for large-area printing of plasmonic pixels is to tile the smallest pixels that still exhibit strong diffractive coupling, as we have done in Figure 4. In doing so, we ensure that the lineshape is sufficiently narrow while allowing edge effects to increase the scattering intensity.

In addition to scaling up to macroscopic devices, displays based on plasmonic pixels can be readily scaled down to extremely small dimensions for applications such as nanobarcodes. In principle, a single nanoparticle could constitute a pixel. Nano-barcodes have been introduced in other nanoscale systems, and include systems such as random groupings of nanowires, ${ }^{51,52}$ phase-changing nanoparticles, ${ }^{53}$ multimetal microrods, ${ }^{54}$ and biomimetic silica-coated polymer fingerprints. ${ }^{55}$ The fabrication process for nanobarcodes must be either randomizable for individual product tracking, or sufficiently reproducible for repeated labeling. The dominant concern for nanobarcodes in this second category would be color control through reproducible, sharp plasmon resonances at the individual pixel level. Interestingly, although many different nanotechnology-based approaches have been applied to either displays or nanobarcodes, few have demonstrated the versatility of the system we have demonstrated, where the same design can be applied to both large- and small-scale applications.

\section{Conclusions}

Our study shows that aluminum nanorod array-based plasmonic pixels can be designed with a spectrally narrow, vivid monochromatic color response, and can be switched on and off using an LCDbased active device geometry. These properties suggest that these pixels may have significant potential for display technologies. These pixels also push the boundaries of the smallest display resolution, with 
near optimal pixel sizes of approximately $5 \mu \mathrm{m}$, compatible with near-eye displays. ${ }^{56}$ The significant color control of these pixels at even smaller pixel sizes lend themselves to applications such as nanobarcodes. ${ }^{57}$ Our aluminum plasmonic pixel color gamut is furthermore comparable to that of the SRGB color gamut, with blue and green colors exceeding SRGB chromaticities. This work provides practical strategies that can be straightforwardly implemented for highly manufacturable, plasmonic pixel color display technology. 


\section{Materials and Methods}

\section{Theoretical Model}

The theoretical calculations are performed using a coupled dipole model. ${ }^{36,48,50,58}$ Briefly, we describe the nanorods as point dipoles with an anisotropic polarizability $\boldsymbol{\alpha}=\alpha_{\mathrm{xx}} \hat{\mathbf{x}} \hat{\mathbf{x}}+\alpha_{\mathrm{yy}} \hat{\mathbf{y}} \hat{\mathbf{y}}+\alpha_{\mathrm{zz}} \hat{\mathbf{z}} \hat{\mathbf{z}}$, which we obtain from a Finite Element Method (FEM) simulation performed using the commercial software COMSOL Multiphysics. Upon illumination with an external field, the dipole induced in each nanorod can be written as

$$
\mathbf{p}_{i}=\boldsymbol{\alpha}\left(\mathbf{E}\left(\mathbf{r}_{i}\right)+\sum_{j} \mathbf{G}_{i j} \mathbf{p}_{j}\right)
$$

where $\mathbf{G}_{i j}=\left[k^{2}+n^{-2} \nabla \nabla\right] \exp \left(i k n\left|\mathbf{r}_{i}-\mathbf{r}_{j}\right|\right) /\left|\mathbf{r}_{i}-\mathbf{r}_{j}\right|$ is the dipole-dipole interaction tensor, $\mathbf{r}_{i}$ are the vectors with the nanorod positions, $\mathbf{E}(\mathbf{r})=\mathbf{E}_{\mathbf{0}} e^{i n \mathbf{k} \cdot \mathbf{r}}$ is the external field with amplitude $\mathbf{E}_{\mathbf{0}}, \mathbf{k}$ is the light wavevector in vacuum, and $n$ is the refractive index of the medium surrounding the nanorod array. The self-consistent induced dipole can be obtained by solving this system of equations as

$$
\mathbf{p}_{i}=\sum_{j}[\mathbf{1}-\boldsymbol{\alpha G}]_{i j}^{-1} \boldsymbol{\alpha E}\left(\mathbf{r}_{j}\right)
$$

Once the induced dipole is known, the power scattered by the ensemble is given by

$$
P=\frac{c n k^{4}}{2 \pi} \operatorname{Re} \sum_{i, j} \int d \Omega \mathrm{e}^{i k n\left(\mathbf{r}_{i}-\mathbf{r}_{j}\right) \cdot \hat{\mathbf{s}}}\left[\mathbf{p}_{i} \cdot \mathbf{p}_{j}^{*}-\left(\mathbf{p}_{i} \cdot \hat{\mathbf{s}}\right)\left(\mathbf{p}_{j}^{*} \cdot \hat{\mathbf{s}}\right)\right]
$$

Here, $\hat{\mathbf{s}}$ is the unit vector in the direction of emission, and the angular integral is performed over the angles compatible with the numerical aperture of the detectors.

In addition to finite arrays, we study the case of an infinite array to obtain deeper insight into the behavior of the plasmonic pixel. Calculations for an infinite array require a slightly different approach. Specifically, we write the dipole induced in each nanorod as

$$
\mathbf{p}_{i}=\mathbf{p}_{\mathbf{k}_{\|}} e^{i \mathbf{k}_{\|} \cdot \mathbf{R}_{i}}
$$


where $\mathbf{k}_{\|}$is the component of the wave vector of the incident field parallel to the array, and $\mathbf{R}_{i}=$ $\left(x_{i}, y_{i}\right)$ is the nanorod position on the array plane. Using this expression, the dipole amplitude can be written as

$$
\mathbf{p}_{\mathbf{k}_{\|}}=\alpha \mathbf{E}_{0}+\alpha g\left(\mathbf{k}_{\|}\right) \mathbf{p}_{\mathbf{k}_{\|}},
$$

where $\boldsymbol{g}\left(\mathbf{k}_{\|}\right)=\sum_{j \neq i} \mathbf{G}_{i j} e^{-i \mathbf{k}_{\|} \cdot\left(\mathbf{R}_{i}-\mathbf{R}_{j}\right)}$. This quantity, which is commonly referred to as the lattice sum, contains the information of the response of the array and therefore determines the properties of the lattice resonances. ${ }^{48,50,58}$ Indeed, a lattice resonance exists whenever $\boldsymbol{g}\left(\mathbf{k}_{\|}\right)$diverges. The wavelength for this resonance condition is given by eq. (2). In our particular geometry these vectors can be obtained from the generalized version of eq. (3):

$$
\mathbf{g}_{(i, j)}=2 \pi\left[\frac{i}{D_{x}} \hat{\mathbf{x}}+\left(\frac{j}{D_{y}}-\frac{i}{2 D_{y}}\right) \hat{\mathbf{y}}\right]
$$

and therefore the wavelength of the lattice resonances are

$$
\lambda_{(i, j)}=\frac{-b+\sqrt{b^{2}-4 a c}}{2 a},
$$

with

$$
\begin{gathered}
\mathrm{a}=\frac{i^{2}}{D_{x}^{2}}+\frac{j^{2}}{D_{y}^{2}}+\frac{i^{2}}{4 D_{y}^{2}}-\frac{i j}{D_{y}^{2}}, \\
\mathrm{~b}=-2 n \sin \theta_{\text {incident }}\left(\frac{i}{D_{x}} \cos \phi_{\text {incident }}+\frac{j}{D_{y}} \sin \phi_{\text {incident }}-\frac{i}{2 D_{y}} \sin \phi_{\text {incident }}\right), \\
c=-n^{2} \cos ^{2} \theta_{\text {incident }} .
\end{gathered}
$$


Finally, to calculate the scattering spectrum we solve the equation for the dipole amplitude $\mathbf{p}_{\mathbf{k}_{\|}}$. This can be done as follows:

$$
\mathbf{p}_{\mathbf{k}_{\|}}=\left[1-\alpha g\left(\mathbf{k}_{\|}\right)\right]^{-1} \boldsymbol{\alpha} \mathbf{E}_{\mathbf{0}}
$$

Once the induced dipole is known, the power scattered per nanorod is given by

$$
\frac{P}{N}=\frac{2 \pi c}{A n} k^{2} \sum_{\mathbf{g}}\left[\left|\mathbf{p}_{\mathbf{k}_{\|}}\right|^{2}-\left|\hat{\mathbf{s}} \cdot \mathbf{p}_{\mathbf{k}_{\|}}\right|^{2}\right],
$$

where $A$ is the area of the unit cell and the summation runs over reciprocal lattice vectors for which $\widehat{\mathbf{s}}=\left(\hat{\mathbf{s}}_{\|}, \hat{\mathbf{s}}_{\perp}\right)$ with $\hat{\mathbf{s}}_{\|}=\left(\mathbf{k}_{\|}-\mathbf{g}\right) / n k$, lies within the collection cone.

\section{Sample Preparation}

An ITO coated clean glass slide (120 nm thick ITO, Sheet Resistance: 8-12 ohm; Delta Technologies Ltd) was spin-coated with a high resolution positive e-beam resist (50\% by volume of PMMA 495 A4 and $50 \%$ by volume of PMMA 495 A2; MicroChem) and baked at $180^{\circ} \mathrm{C}$ for $90 \mathrm{~s}$. The substrate was then patterned in a scanning electron microscope (FEI QUANTA 650 equipped with a beam blanker and associated with the Nabity NPGS software) and developed in a 1:3 v/v solution of MIBK:IPA for $30 \mathrm{~s}$. Thirty five $\mathrm{nm}$ of aluminum was then deposited onto the substrate via e-beam evaporation, at a rate of $0.8-0.9 \AA / s$ and a base pressure of approximately $2.2 \times 10^{-7}$ torr. The low base pressure and slow evaporation rate ensured minimal oxygen content in the chamber resulting in an aluminum oxide content of 9\%. ${ }^{59}$ Lift-off was carried out by soaking the sample in acetone for approximately $15 \mathrm{~h}$, followed by gentle rinsing with fresh acetone and drying under a flow of nitrogen gas. After SEM characterization using the same electron microscope, the sample was spincoated with PI solution (SE3510; Nissan Chemical) and baked at $180^{\circ} \mathrm{C}$ for $45 \mathrm{~min}$. and cooled to room temperature. Unless otherwise noted, all pixel footprints are $5 \mu \mathrm{m} \times 5 \mu \mathrm{m}$.

To prepare a LCD device, a plasmonic pixel sample was first prepared as noted above. An additional substrate was also prepared by cleaning an ITO slide and spin coating it with PI and baking, following the aforementioned procedure. Micro grooves for alignment of the liquid crystal were created by gently rubbing the PI coated surface of both samples unidirectionally with a lens cloth. For the slide with the 
pixels, the rubbing direction was chosen to be parallel to the nanorods' long axis. In this way, the alignment of the liquid crystal nematic director and therefore the main optical axis of the birefringent liquid crystal were predefined. A silica beads solution (Microsil microspheres, SSO6N, $6 \mu \mathrm{m}$ in diameter) was then spin-coated onto the PI side of the slide containing the pixels, avoiding the pixel area, to control the device thickness. The two substrates were then assembled into a liquid crystal cell so that the PI surfaces face each other, with rubbing directions perpendicular to each other to promote a twisted nematic conformation when no voltage is applied. Clear epoxy (Norland Products Inc., UVS91) was used to glue two sides of this cell. The epoxy was then cured with a UV lamp (Spectronics, EA-160) operating at $365 \mathrm{~nm}$ for 20 minutes. Capillary action was used to insert the liquid crystal 5CB (Tokyo Chemical, C1550), at $60^{\circ} \mathrm{C}$ in its isotropic phase, into the prepared cell. The filled liquid crystal cell was allowed to cool to room temperature over approximately 1.5 hours before sealing the two open sides with the same epoxy glue. Finally, a conductive silver epoxy (Chemtronics, CW2400) was used to connect copper wires to the ITO surface of each slide.

\section{SEM and Optical Characterization}

SEM imaging of the nanorod size and array spacing within a pixel was performed with the same SEM used for lithographic patterning of the sample (FEI QUANTA 650), where the images were acquired prior to optical characterization to preserve the integrity of the PI layer. Multiple iterations of the same pattern were prepared in the same region of a sample so that SEM characterization could be performed on nearby but identically prepared sets of pixels, not on the pixels that were later characterized optically to avoid damage to the pixels by SEM imaging. Samples were imaged at a working distance of $10 \mathrm{~mm}$ and with 100,000x magnification for automated size analysis carried out in Matlab. Scattering images and spectral measurements were taken by placing the sample on an inverted microscope (Axiovert 200; Zeiss) with the pixel side facing down towards the objective (50x, NA =0.8, EC Epiplan-NEOFLUAR; Zeiss). P-polarized white light excitation from a tungsten lamp (Newport) mounted in a cage system (Thorlabs) was coupled to the inverted substrate using an equilateral prism (Thorlabs). Spectra from individual pixels were collected by passing the scattered light through a $50-\mu \mathrm{m}$ pinhole at the first image plane of the microscope to a spectrometer (Acton SP2150i; Princeton Instruments) and CCD camera (PIXIS 400BR; Princeton Instruments). A center wavelength of $600 \mathrm{~nm}$ was used for all spectra. Each spectrum represents an average over three spectra taken. All spectra were background corrected by 
subtracting a background spectrum recorded with the same exposure time at a position with no pixels present. Each spectrum was further divided by a transmission spectrum of the lamp through the same substrate at a position on the sample where no pixels were present.

The integrated intensities of the pixels of varying sizes were obtained by imaging the pixels under the same excitation and collection geometry described above, but with the following modifications. The pinhole was removed from the detection path, and the grating was set to the zero order reflection (imaging mode). The exposure time of the CCD camera was $2 \mathrm{~s}$ for all acquisitions. Image analysis was performed in Matlab by subtracting the average background intensity from the whole image, then summing the intensity from the resulting pixel image. This integrated intensity is divided by the number of nanorods per pixel. Multiple images of the same type of Al-pixel were taken using the same exposure time, to build up statistics, as further explained in the Supplemental Information.

\section{Out-of-Microscope Digital Camera Images}

The sample was mounted vertically and white light was coupled in from the side of the slide using a glass fiber bundle (Newport, part no. 77538) with a rectangular output slit. The white light entered the slide and propagated within the slide along the long axes of the nanorods in the pixels. A polarizer was placed between the fiber and the sample edge so that the excitation light was initially polarized perpendicular to the glass substrates that acted as a waveguide. Excitation angles and polarization were then defined by total internal reflection. Digital camera images were taken using a Canon EOS Rebel T2i digital single lens reflex camera with ISO set to 100 and various exposure times. The camera was mounted in a slider situated on a semicircular track (Movo Photo CTS500, 51" Length) to obtain images from various angles at the same viewing distance. When this same slide was incorporated into a liquid crystal device, the liquid crystal was placed in contact with the glass slide, which also changed the excitation geometry slightly. Therefore, a comparison of these images of macroscopic pixels with and without liquid crystal is not quantitative. A polarizer was also placed between the camera and the sample. For single-substrate samples without the liquid crystal, the pixels were viewed through the glass surface because the pixels were bright enough to create a reflection on the glass surface when viewed through the PI surface, making it difficult to focus the camera. The LCD devices were imaged through the liquid crystal, where the PI coating of the pixel was facing the camera. The pixels were also viewed under 
the same conditions through an additional small sheet of $80^{\circ}$ optical diffuser, commercially available from Luminit.

\section{Supporting Information}

Supporting information contains a discussion and videos of the viewing angle dependence of pixel color with and without an optical diffuser, an estimate of the efficiency of a plasmonic pixel, further detail on the color reproducibility for varying sizes of pixels, and a video of active switching of the plasmonic pixels. This material is available free of charge via the internet at http://pubs.acs.org.

\section{Corresponding Author}

To whom correspondence should be addressed:

Stephan Link Department of Chemistry

Rice University

6100 Main Street MS - 60

Houston, TX 77005

Tel: 713-348-4561

Email: slink@rice.edu

\section{Author Contributions}

This manuscript was written through contributions of all authors. All authors have given approval to the final version of the manuscript.

\section{Acknowledgements}

The authors thank Dr. Nicholas King, Dr. Mark Knight, and Dr. Wei-Shun Chang for their helpful discussion. This work was funded by Robert A. Welch Foundation Grants C-1220, C-1222, and C-1664 and Office of Naval Research Grant N00014-10-0989. A.M. acknowledges financial support from the 
Welch foundation through the J. Evans Attwell-Welch Postdoctoral Fellowship Program of the Smalley Institute of Rice University, and the Department of Physics and Astronomy and the College of Arts and Sciences of the University of New Mexico. J.O. acknowledges support from the National Science Foundation through Graduate Research Fellowship 0940902. 


\section{References}

1. Dean, N., Colouring at the nanoscale. Nat. Nanotechnol. 2015, 10, 15-16.

2. Link, S.; Mohamed, M. B.; El-Sayed, M. A., Simulation of the Optical Absorption Spectra of Gold Nanorods As a Function of Their Aspect Ratio and the Effect of the Medium Dielectric Constant. J. Phys. Chem. B 1999, 103, 3073-3077.

3. Zoric, I.; Zach, M.; Kasemo, B.; Langhammer, C., Gold, Platinum, and Aluminum Nanodisk Plasmons: Material Independence, Subradiance, and Damping Mechanisms. ACS Nano 2011, 5, 2535-2546.

4. Mock, J. J.; Barbic, M.; Smith, D. R.; Schultz, D. A.; Schultz, S., Shape Effects in Plasmon Resonance of Individual Colloidal Silver Nanoparticles. J. Chem. Phys. 2002, 116, 6755-6759.

5. Anikeeva, P. O.; Halpert, J. E.; Bawendi, M. G.; Bulović, V., Quantum Dot Light-Emitting Devices with Electroluminescence Tunable Over the Entire Visible Spectrum. Nano Lett. 2009, 9, 2532-2536.

6. Sun, Q.; Wang, Y. A.; Li, L. S.; Wang, D.; Zhu, T.; Xu, J.; Yang, C.; Li, Y., Bright, Multicoloured LightEmitting Diodes Based on Quantum Dots. Nat. Photon. 2007, 1, 717-722.

7. Roberts, A. S.; Pors, A.; Albrektsen, O.; Bozhevolnyi, S. I., Subwavelength Plasmonic Color Printing Protected for Ambient Use. Nano Lett. 2014, 14, 783-787.

8. Kumar, K.; Duan, H.; Hegde, R. S.; Koh, S. C. W.; Wei, J. N.; Yang, J. K. W., Printing Colour at the Optical Diffraction Limit. Nat. Nanotechnol. 2012, 7 , 557-561.

9. Ozaki, M.; Kato, J.-i.; Kawata, S., Surface-Plasmon Holography With White-Light Illumination. Science 2011, 332 (6026), 218-220.

10. Hsu, C. W.; Zhen, B.; Qiu, W.; Shapira, O.; DeLacy, B. G.; Joannopoulos, J. D.; Soljačić, M., Transparent Displays Enabled by Resonant Nanoparticle Scattering. Nat. Commun. 2014, 5, 3152.

11. Gomez, D. E.; Vernon, K. C.; Mulvaney, P.; Davis, T. J., Coherent Superposition of Exciton States in Quantum Dots Induced by Surface Plasmons. Appl. Phys. Lett. 2010, 96, 073108.

12. Tan, S. J.; Zhang, L.; Zhu, D.; Goh, X. M.; Wang, Y. M.; Kumar, K.; Qiu, C.-W.; Yang, J. K. W., Plasmonic Color Palettes for Photorealistic Printing with Aluminum Nanostructures. Nano Lett. 2014, 14, 40234029.

13. Clausen, J. S.; Højlund-Nielsen, E.; Christiansen, A. B.; Yazdi, S.; Grajower, M.; Taha, H.; Levy, U.; Kristensen, A.; Mortensen, N. A., Plasmonic Metasurfaces for Coloration of Plastic Consumer Products. Nano Lett. 2014, 14, 4499-4504. 
14. Wen, L.; Chen, Q.; Sun, F.; Song, S.; Jin, L.; Yu, Y., Theoretical Design of Multi-Colored SemiTransparent Organic Solar Cells With Both Efficient Color Filtering and Light Harvesting. Sci. Rep. 2014, 4, 7036.

15. Yokogawa, S.; Burgos, S. P.; Atwater, H. A., Plasmonic Color Filters for CMOS Image Sensor Applications. Nano Lett. 2012, 12 (8), 4349-4354.

16. Shrestha, V. R.; Lee, S.-S.; Kim, E.-S.; Choi, D.-Y., Aluminum Plasmonics Based Highly Transmissive Polarization-Independent Subtractive Color Filters Exploiting a Nanopatch Array. Nano Lett. 2014, $14,6672-6678$.

17. Burgos, S. P.; Yokogawa, S.; Atwater, H. A., Color Imaging via Nearest Neighbor Hole Coupling in Plasmonic Color Filters Integrated Onto a Complementary Metal-Oxide Semiconductor Image Sensor. ACS Nano 2013, 7, 10038-10047.

18. Formica, N.; Mantilla-Perez, P.; Ghosh, D. S.; Janner, D.; Chen, T. L.; Huang, M.; Garner, S.; Martorell, J.; Pruneri, V., An ITO-free Polymer Solar Cell on Flexible Glass. ACS Appl. Mater. Interfaces 2015, 7, 4541-4548.

19. Knight, M. W.; Sobhani, H.; Nordlander, P.; Halas, N. J., Photodetection With Active Optical Antennas. Science 2011, 332, 702-704.

20. Li, Z.; Butun, S.; Aydin, K., Large-Area, Lithography-Free Super Absorbers and Color Filters at Visible Frequencies Using Ultrathin Metallic Films. ACS Photonics 2015, 2, 183-188.

21. Si, G.; Zhao, Y.; Liu, H.; Teo, S.; Zhang, M.; Jun Huang, T.; Danner, A. J.; Teng, J., Annular Aperture Array Based Color Filter. Appl. Phys. Lett. 2011, 99, 033105.

22. Xu, T.; Wu, Y.-K.; Luo, X.; Guo, L. J., Plasmonic Nanoresonators for High-Resolution Colour Filtering and Spectral Imaging. Nat. Commun. 2010, 1, 59.

23. Chen, Q.; Cumming, D. R. S., High Transmission and Low Color Cross-talk Plasmonic Color Filters Using Triangular-Lattice Hole Arrays in Aluminum Films. Opt. Express 2010, 18, 14056-14062.

24. Frey, L.; Parrein, P.; Raby, J.; Pellé, C.; Hérault, D.; Marty, M.; Michailos, J., Color Filters Including Infrared Cut-off Integrated on CMOS Image Sensor. Opt. Express 2011, 19, 13073-13080.

25. Zheng, B. Y.; Wang, Y.; Nordlander, P.; Halas, N. J., Color-Selective and CMOS-Compatible Photodetection Based on Aluminum Plasmonics. Adv. Mater. 2014, 26, 6318-6323.

26. Lozano, G.; Louwers, D. J.; Rodríguez, S. R.; Murai, S.; Jansen, O. T.; Verschuuren, M. A.; Gómez Rivas, J., Plasmonics for Solid-state Lighting: Enhanced Excitation and Directional Emission of Highly Efficient Light Sources. Light: Science \& Applications 2013, 2, e66.

27. Wang, Y. In Surface Plasmon Tunable Filters and Flat Panel Display Device, 1999; pp 69-72. 
28. Ellenbogen, T.; Seo, K.; Crozier, K. B., Chromatic Plasmonic Polarizers for Active Visible Color Filtering and Polarimetry. Nano Lett. 2012, 12, 1026-31.

29. King, N. S.; Liu, L.; Yang, X.; Cerjan, B.; Everitt, H. O.; Nordlander, P.; Halas, N. J., Fano Resonant Aluminum Nanoclusters for Plasmonic Colorimetric Sensing. ACS Nano 2015 Article ASAP.

30. Liu, H.-W.; Lin, F.-C.; Lin, S.-W.; Wu, J.-Y.; Chou, B.-T.; Lai, K.-J.; Lin, S.-D.; Huang, J.-S., SingleCrystalline Aluminum Nanostructures on a Semiconducting GaAs Substrate for Ultraviolet to NearInfrared Plasmonics. ACS Nano 2015, 9, 3875-3886.

31. Inoue, D.; Miura, A.; Nomura, T.; Fujikawa, H.; Sato, K.; Ikeda, N.; Tsuya, D.; Sugimoto, Y.; Koide, Y., Polarization Independent Visible Color Filter Comprising an Aluminum Film with Surface-plasmon Enhanced Transmission Through a Subwavelength Array of Holes. Appl. Phys. Lett. 2011, 98, 093113.

32. Cheng, F.; Gao, J.; Stan, L.; Rosenmann, D.; Czaplewski, D.; Yang, X., Aluminum Plasmonic Metamaterials for Structural Color Printing. Opt. Express 2015, 23, 14552-14560.

33. Shen, Y.; Rinnerbauer, V.; Wang, I.; Stelmakh, V.; Joannopoulos, J. D.; Soljačić, M., Structural Colors from Fano Resonances. ACS Photonics 2015, 2, 27-32.

34. Gao, L.; Zhang, Y.; Zhang, H.; Doshay, S.; Xie, X.; Luo, H.; Shah, D.; Shi, Y.; Xu, S.; Fang, H. et. al. Optics and Nonlinear Buckling Mechanics in Large-Area, Highly Stretchable Arrays of Plasmonic Nanostructures. ACS Nano 2015, Article ASAP.

35. Franklin, D.; Chen, Y.; Vazquez-Guardado, A.; Modak, S.; Boroumand, J.; Xu, D.; Wu, S.-T.; Chanda, D., Polarization-Independent Actively Tunable Colour Generation on Imprinted Plasmonic Surfaces. Nat. Commun. 2015, 6, 7337.

36. Olson, J.; Manjavacas, A.; Liu, L.; Chang, W.-S.; Foerster, B.; King, N. S.; Knight, M. W.; Nordlander, P.; Halas, N. J.; Link, S., Vivid, Full-color Aluminum Plasmonic Pixels. Proc. Natl. Acad. Sci. U. S. A. 2014, $111,14348-14353$.

37. Hua, F.; Sun, Y.; Gaur, A.; Meitl, M. A.; Bilhaut, L.; Rotkina, L.; Wang, J.; Geil, P.; Shim, M.; Rogers, J. A.; et. al. Polymer Imprint Lithography with Molecular-Scale Resolution. Nano Lett. 2004, 4, 24672471.

38. Malyarchuk, V.; Hua, F.; Mack, N. H.; Velasquez, V. T.; White, J. O.; Nuzzo, R. G.; Rogers, J. A., High Performance Plasmonic Crystal Sensor Formed by Soft Nanoimprint Lithography. Opt. Express 2005, 13, 5669-5675.

39. Shen, Y.; Yao, L.; Li, Z.; Kou, J.; Cui, Y.; Bian, J.; Yuan, C.; Ge, H.; Li, W.-D.; Wu, W.; Chen, Y., Double Transfer UV-curing Nanoimprint Lithography. Nanotechnology 2013, 24, 465304. 
40. Audesirk, H. A.; Warren, E. L.; Ku, J.; Lewis, N. S., Ordered Silicon Microwire Arrays Grown from Substrates Patterned Using Imprint Lithography and Electrodeposition. ACS Appl. Mater. Interfaces 2015, 7, 1396-1400.

41. Suresh, V.; Huang, M. S.; Srinivasan, M. P.; Krishnamoorthy, S., Macroscopic High Density Nanodisc Arrays of Zinc Oxide Fabricated by Block Copolymer Self-assembly Assisted Nanoimprint Lithography. J. Mater. Chem. 2012, 22, 21871-21877.

42. Gao, L.; Shigeta, K.; Vazquez-Guardado, A.; Progler, C. J.; Bogart, G. R.; Rogers, J. A.; Chanda, D., Nanoimprinting Techniques for Large-area Three-dimensional Negative Index Metamaterials with Operation in the Visible and Telecom Bands. ACS Nano 2014, 8, 5535-5542.

43. Vala, M.; Homola, J., Flexible Method Based on Four-beam Interference Lithography for Fabrication of Large Areas of Perfectly Periodic Plasmonic Arrays. Opt. Express 2014, 22, 18778-18789.

44. Kusaka, K.; Kurosawa, H.; Ohno, S.; Sakaki, Y.; Nakayama, K.; Moritake, Y.; Ishihara, T., Waveguidemode Interference Lithography Technique for High Contrast Subwavelength Structures in the Visible Region. Opt. Express 2014, 22, 18748-18756.

45. Ma, Y.; Sun, N.; Zhang, R.; Guo, L.; She, Y.; Zheng, J.; Ye, Z., Integrated Color Filter and Polarizer Based on Two-Dimensional Superimposed Nanowire Arrays. J. Appl. Phys. 2014, 116, 044314.

46. Birgit, P.; Andreas, L.; Eugenie, K.; Christian, D.; Yasin, E., Sub-10 nm Patterning Using EUV Interference Lithography. Nanotechnology 2011, 22, 375302.

47. Ekinci, Y.; Solak, H. H.; Löffler, J. F., Plasmon Resonances of Aluminum Nanoparticles and Nanorods. J. Appl. Phys. 2008, 104, 083107.

48. Teperik, T. V.; Degiron, A., Design Strategies to Tailor the Narrow Plasmon-photonic Resonances in Arrays of Metallic Nanoparticles. Phys. Rev. B 2012, 86, 245425.

49. Norman, J. C.; DeJarnette, D. F.; Roper, D. K., Polylogarithm-Based Computation of Fano Resonance in Arrayed Dipole Scatterers. J. Phys. Chem. C 2014, 118, 627-634.

50. García de Abajo, F. J., Colloquium: Light Scattering by Particle and Hole Arrays. Rev. Mod. Phys. 2007, 79, 1267-1290.

51. Kim, J.; Yun, J. M.; Jung, J.; Song, H.; Kim, J.-B.; Ihee, H., Anti-Counterfeit Nanoscale Fingerprints Based on Randomly Distributed Nanowires. Nanotechnology 2014, 25, 155303.

52. Gudiksen, M. S.; Lauhon, L. J.; Wang, J.; Smith, D. C.; Lieber, C. M., Growth of Nanowire Superlattice Structures for Nanoscale Photonics and Electronics. Nature 2002, 415, 617-620.

53. Duong, B.; Liu, H.; Ma, L.; Su, M., Covert Thermal Barcodes Based on Phase Change Nanoparticles. Sci. Rep. 2014, 4, 5170. 
54. Nicewarner-Pena, S. R.; Freeman, R. G.; Reiss, B. D.; He, L.; Pena, D. J.; Walton, I. D.; Cromer, R.; Keating, C. D.; Natan, M. J., Submicrometer Metallic Barcodes. Science 2001, 294, 137-141.

55. Bae, H. J.; Bae, S.; Park, C.; Han, S.; Kim, J.; Kim, L. N.; Kim, K.; Song, S.-H.; Park, W.; Kwon, S., Biomimetic Microfingerprints for Anti-Counterfeiting Strategies. Adv. Mater. 2015, 27, 2083-2089.

56. Gross, H.; Blechinger, F.; Achtner, B., CH 36: Human Eye. In Handbook of Optical Systems, Volume 4, Survey of Optical Instruments, John Wiley \& Sons, Inc.: 2008.

57. Echegoyen, Y., Nano-developments for Food Packaging and Labeling Applications. In Nanotechnologies in Food and Agriculture, Rai, M.; Ribeiro, C.; Mattoso, L.; Duran, N., Eds. Springer International Publishing: 2015; pp 141-166.

58. Zhao, L.; Kelly, K. L.; Schatz, G. C., The Extinction Spectra of Silver Nanoparticle Arrays: Influence of Array Structure on Plasmon Resonance Wavelength and Width. J. Phys. Chem. B 2003, 107, 73437350.

59. Knight, M. W.; King, N. S.; Liu, L.; Everitt, H. O.; Nordlander, P.; Halas, N. J., Aluminum for Plasmonics. ACS Nano 2013, 8, 834-840. 
Graphical Table of Contents

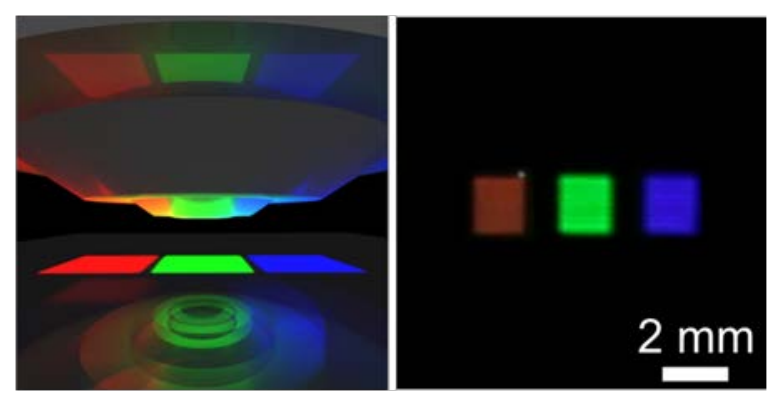

